\title{
Cardiac MRI detection of a rare case of familial cardiac amyloidosis (Ser23Asn): case report with literature review
}

This article was published in the following Dove Press journal:

Reports in Medical Imaging

8 December 2010

Number of times this article has been viewed

\section{Joseph Daoko \\ Yaser Elnahar \\ Karim El Kersh \\ Naser Mohammad \\ Fayez Shamoon}

St Michael's Medical Center

Newark, NJ, USA
Correspondence: Yaser Elnahar St Michael's Medical Center, I I I Central Avenue, Newark, NJ 07102, USA

Tel +l 732763 I862

Email yaserelnahar@hotmail.com

\begin{abstract}
Cardiac amyloidosis is a rare disease that has significant sequelae if undetected and treated early. We report a case of a rare genetic mutation that leads to cardiac amyloidosis. This is a case of a 41-year-old Peruvian male without any known past medical history but with a family history of sudden death. The patient complained of episodic left-sided chest pain for 1 month that was associated with shortness of breath. A subsequent admission and workup for coronary artery disease was negative, but an echocardiogram showed global left ventricular hypokinesia. Cardiac magnetic resonance imaging (MRI) was performed which was suggestive of cardiac amyloidosis. A subsequent abdominal fat biopsy and bone marrow biopsy failed to show evidence of amyloidosis. However, the endomyocardial biopsy was consistent with cardiac amyloidosis. Familial cardiac disease was suspected because of the family history of sudden death. As such, genetic testing was performed which yielded a very rare genetic mutation corresponding to amino acid 23 of the transthyretin protein. A review of the literature shows that this gene mutation (AGT23AAT, gene sequence; Ser23Asn, protein sequence) has been described only once previously. This case is unique in that the patient's amyloidosis was limited to the heart, without involvement of any other organ.
\end{abstract}

Keywords: amyloidosis, mutation, cardiac disease, genetics, diagnosis

\section{Background}

Traditional means of diagnosing amyloidosis rely on a good history in conjunction with a fat pad biopsy and/or bone marrow biopsy. This case is interesting in that both biopsies were negative in the setting of a magnetic resonance imaging (MRI) finding suggestive of cardiac amyloidosis (CA). Subsequent genetic testing yielded a very rare gene mutation that has been reported only once in the literature. We report the presentation, workup, and outcome of this rare disease in addition to a literature review of the subject.

\section{Case presentation}

We present a case of a 41-year-old Peruvian male without any known past medical history but with a family history of sudden cardiac death (SCD) (mother and two brothers) due to presumed hypertrophic cardiomyopathy (HCM) as informed in Peru. He denied smoking, and alcohol, and drug use. The patient complained of recurrent left-sided chest pain for the last month. He stated that the pain radiated to the left shoulder and was associated with shortness of breath. He denied dizziness, palpitations, diaphoresis, and syncope. He had no loss of appetite, no diarrhea, and no weight loss. He denied symptoms of peripheral neuropathy. His physical exam showed 
the following: 1) normal vital signs without evidence of orthostatic hypotension, 2) normal fundoscopic evaluation, 3) cardiac exam showed normal $\mathrm{S}_{1}$ and $\mathrm{S}_{2}$ without displacement of the point of maximum impulse, 4) the lungs were clear to auscultation bilaterally without any evidence of wheezing, rhonchi, or rales, 5) the abdomen was soft, nontender/ distended with equal bowel sounds, 6) the distal pulses were equal, 7) his skin was normal without any rashes, and 8) the neurological exam was normal. The electrocardiogram (EKG) showed first-degree atrioventricular block, left atrial enlargement, and right bundle branch block without any evidence of left ventricular hypertrophy by voltage criteria. A low voltage was not present. The admission labs were all within normal limits including a negative protein in the urine analysis. The patient underwent a nuclear stress test that showed perfusion defects in the inferior and lateral walls. Two-dimensional (2D) EKG showed moderate concentric left ventricular hypertrophy, and moderate pericardial effusion, without evidence of hypertrophic obstructive cardiomyopathy (HOCM); the remainder of the 2D EKG was normal. Left heart catheterization rendered normal coronaries and global left ventricular hypokinesia. Due to the family history of sudden death and the abnormal 2D EKG, a cardiac MRI was performed which was consistent with CA (Figures 1 and 2). A subsequent abdominal fat biopsy failed to show evidence of amyloidosis. Moreover, a bone marrow biopsy was performed as the former was negative for amyloidosis. The subsequent endomyocardial biopsy (EMB) yielded interstitial deposit of amorphous eosinophilic material consistent with amyloid (Congo red stain positive with apple-green birefringence by polarization). Due to the high suspicion of familial CA, genetic testing was performed which yielded

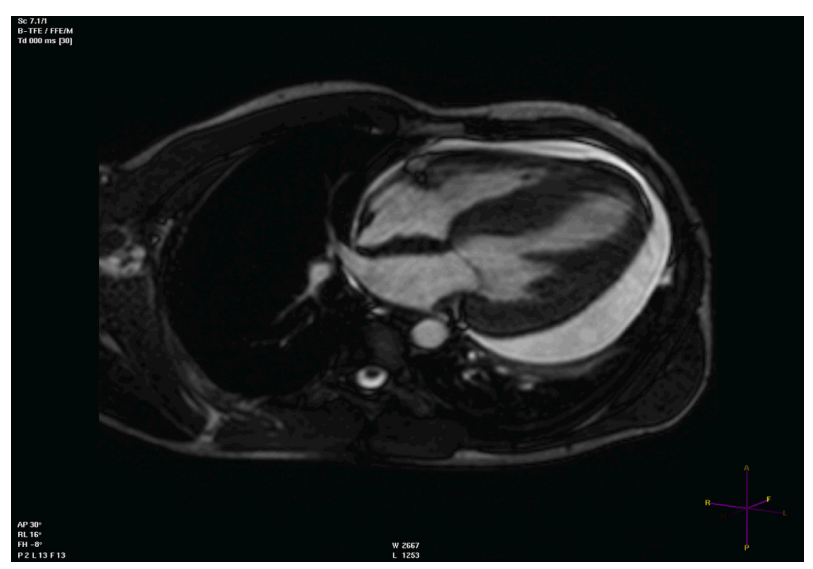

Figure I Four-chamber view showing left ventricular hypertrophy, right ventricular hypertrophy, pericardial effusion, and thickening of the intra-atrial septum.

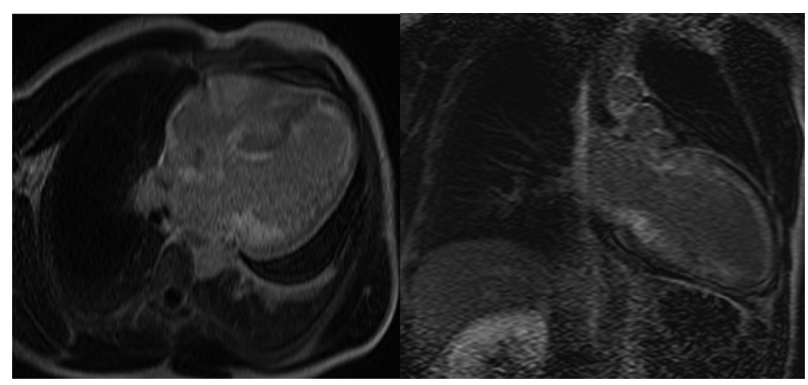

Figure 2 Three-chamber view (left) and two-chamber view (right) showed diffuse enhancement of the endocardium.

a very rare genetic mutation corresponding to amino acid 23 of the transthyretin (TTR) protein. The patient underwent an implantable defibrillator placement and was referred for liver and heart transplantation. Literature review shows that the mutation (AGT23AAT, gene sequence; Ser23Asn, protein sequence) has been reported only once previously. ${ }^{1}$ This case is unique in that the patient's amyloidosis was limited to the heart, without involvement of any other organ.

\section{CMR imaging}

All images were acquired on a Philips Intera 1.5 Tesla scanner (Philips Healthcare, Amsterdam, The Netherlands) with a phased array receiver coil, during breath holds gated to the EKG. Cine images were acquired in multiple short-axis and long-axis views with a steady-state free precession (SSFP) technique (slice thickness $8 \mathrm{~mm}$, flip angle $60^{\circ}$, repetition time [TR] $3.0 \mathrm{~ms}$, echo time [TE] $1.5 \mathrm{~ms}$ ), and it showed biventricular hypertrophy, thickening of the atrial septum, and pericardial effusion (Figure 1). A gadoliniumbased contrast agent (gadopentetate dimeglumine; Bayer Healthcare Pharmaceuticals, Wayne, NJ) was then administered intravenously in a dose of $0.2 \mathrm{~mL} / \mathrm{kg}$ and contrastenhanced images were acquired in the same views used for cine imaging on average $10 \mathrm{~min}$ after contrast administration (flip angle $15^{\circ}$, TR $3.3 \mathrm{~ms}$, TE $1.1 \mathrm{~ms}$ ); the latter revealed diffuse myocardial late gadolinium enhancement (LGE) (Figure 2).

\section{Discussion and literature review}

To date, there have been numerous identified genetic mutations that led to TTR amyloidosis. This case represents a rare mutation of a TTR amyloidosis that has been reported only once previously. This case is unique in that multiple traditional diagnostic modalities were negative for identifying amyloidosis. The family history of SCD and unexplained cardiomyopathy led to the workup for infiltrative heart disease. The subsequent MRI was essential in leading to 
EMB, which led to the ultimate diagnosis. In the previously reported case, the patient was a 44-year-old Portuguese male who had a 3-year history of unexplained fatigue. ${ }^{1}$ In contrast to our patient, the previously reported case was detected by an abdominal fat biopsy with a subsequent EMB.

Amyloidosis is a multisystemic inflammatory disorder with multiple types. A feature that is uniform among the different types is the amyloidogenic protein. The identity of the amyloidogenic protein varies with the type of amyloidosis. Plasma cell dyscrasia (light-chain immunoglobulin, amyloid light-chain [AL] amyloidosis) as well as TTR amyloidosis share a feature of having cardiac involvement. However, amyloid A (AA) amyloidosis rarely produces clinically apparent heart disease. Cardiac amyloidosis is characterized by interstitial amyloid infiltration leading to thickened cardiac walls and diastolic dysfunction, which eventually results in restrictive cardiomyopathy.

The name 'amyloidosis' is attributed to the pathologist Virchow (1854) who thought that amyloid deposits were cellulose-like due to similar staining properties when exposed to iodine. ${ }^{2}$ We now know that amyloidosis is a disease that is due to extracellular deposition of insoluble polymeric protein fibrils which share a common B-pleated sheet. These amyloid deposits are characterized by their apple-green birefringence under a polarized light (using a Congo red stain) and the presence of rigid, nonbranching fibrils on electron microscopy. ${ }^{3}$ There are at least 25 different human and eight different animal protein precursors of amyloid fibrils. ${ }^{4}$ Despite having heterogeneous structures and functions, all these proteins can generate morphologically indistinguishable amyloid fibrils. ${ }^{5}$ Amyloidosis is classified chemically according to the type of protein in the fibril. The nomenclature is $\mathrm{AX}$, where the capital letter ' $A$ ' stands for amyloidosis and ' $\mathrm{X}$ ' represents the fibril protein. ${ }^{6}$

TTR is a transport protein synthesized mainly in the liver but also in the choroid plexus and in the retinal pigment epithelium. The protein functions as a serum and cerebrospinal fluid carrier of thyroxine and retinol. Normal plasma TTR concentration is $20-40 \mathrm{mg} / \mathrm{dL}(0.20-0.40 \mathrm{mg} / \mathrm{mL})$. TTR is a tetramer of four identical subunits of 127 amino acids each. Transthyretin-associated amyloidosis (ATTR) is a disease caused by dissociation of the TTR tetramer. Once dissociated, the monomers deposit in various organs. This structural instability of the TTR tetramer can occur due to genetic mutations of the gene encoding the TTR protein leading to familial amyloidosis. Mutant-sequence TTR forms the amyloid deposits. ${ }^{7}$ These deposits can be associated with aging, thus leading to senile amyloidosis in which normalsequence TTR (wild-type) forms amyloid deposits. Point mutations in TTR increase the tendency of TTR to form amyloid. Amyloidogenic TTR mutations are inherited as an autosomal-dominant disease with variable penetrance. More than 100 amyloidogenic TTR mutations are known. ${ }^{8}$ With the exception of del122Ile, no deletions or duplications involving TTR as causative of TTR amyloidosis have been reported. Few mutations are not associated with amyloidosis, and some are thought to protect against the deposition of amyloid (suppressor mutations). ${ }^{9}$ TTR stability plays an important role in disease pathogenesis. When compared to wild-type TTR (Figure 3), the amyloidogenic mutant-sequence TTR is less stable, but suppressor mutations (Thr119Met and Arg104His) are more stable. The degree of amyloidogenicity

\section{TTR (Transthyretin)}

\section{Normal-sequence TTR (wild type)}<smiles>C1=CC=CCCCC=C1</smiles>

Results from the pathologic deposition of wild-type TTR, predominantly in the heart. Pathologic deposits are also seen in lungs, blood vessels, and the renal medulla of kidneys. ${ }^{24}$
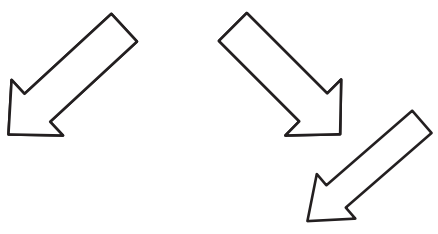

Mutant-sequence TTR
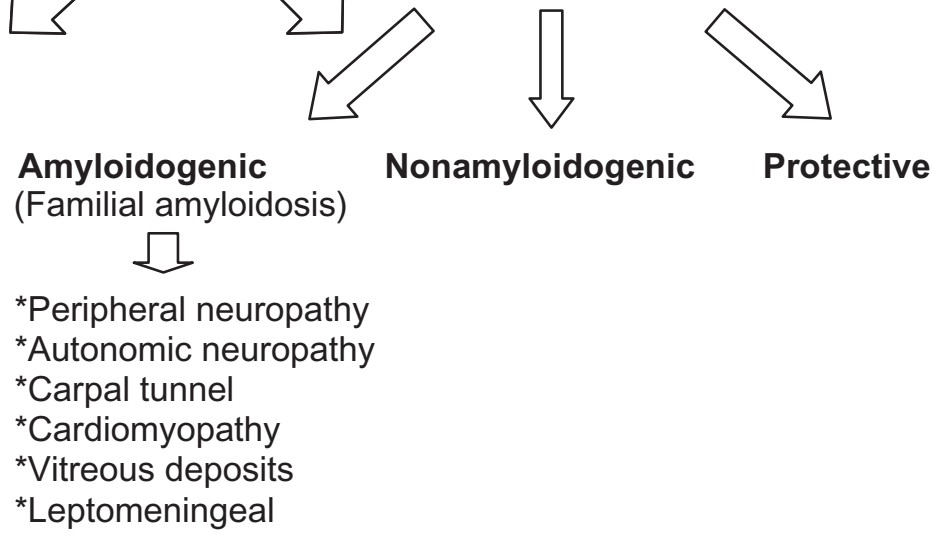

Figure 3 Transthyretin has many mutations, each of which manifests variably. The different TTR gene sequence mutations are outlined above. 
correlates with protein stability, but extremely destabilized TTR usually does not cause severe systemic amyloidosis. This is due to a very low serum concentration as a result of degradation by endoplasmic reticulum of hepatic cells..$^{7,10-12}$ In TTR-associated amyloidosis due to a mutation in the TTR molecule (ATTR), the presence of cardiac involvement varies with the specific mutation, ${ }^{13}$ but cardiac involvement has been reported with more than two-thirds of TTR mutations. Clinical features of familial TTR amyloidosis are variable depending upon the underlying mutation. Organ involvement can include polyneuropathy (motor, sensory, and autonomic), carpal tunnel syndrome, cardiomyopathy, leptomeningeal, and ocular manifestations secondary to vitreous opacities derived from amyloid that may lead to visual impairment. CA has a rapidly progressive course in the case of AL amyloidosis with a median survival of 6 months in untreated patients that present with symptomatic heart failure, ${ }^{14}$ while in systemic senile or familial amyloidosis, the course is often slower. ${ }^{15}$

The symptoms of CA include atrial, atrioventricular, and ventricular conduction disturbances, ventricular and supraventricular arrhythmias, orthostatic symptoms, restrictive cardiomyopathy, angina, and heart failure. CA can progress from an asymptomatic stage to early symptomatic stage with diastolic dysfunction. Reduced systolic function is seen with disease progression. In patients with AL amyloidosis, elevated serum brain natriuretic peptide (BNP) is seen before the onset of clinical heart failure and is considered a marker of cardiac involvement. In one study, a BNP value within normal limit made the likelihood of CA close to $0 \% .{ }^{16}$ Angina is another common manifestation and is secondary to amyloid deposition in the coronary arteries. The deposits typically involve small intramyocardial blood vessels, and as a result coronary angiography is typically normal or shows only minor abnormalities. ${ }^{17,18} \mathrm{CA}$ can also present with syncope or presyncope which indicates severe restrictive cardiomyopathy; however, other factors may play a role such as postural hypotension secondary to associated autonomic dysfunction. ${ }^{19}$ Asymmetric septal hypertrophy is more common in patients with familial amyloidosis than in AL amyloidosis and TTR mutation. Further workup should be considered in cases of HCM that are not explained by mutations in sarcomeric protein genes. ${ }^{20}$ Finally, patients with $\mathrm{CA}$ are prone to conduction abnormalities especially in the His-Purkinje system and prolongation of the HV interval. The aforementioned is common even in the presence of a narrow QRS complex. ${ }^{21}$

Currently, the noninvasive tests that are employed in the diagnosis of amyloidosis are EKG and echocardiography. ${ }^{22}$
The EKG has limited diagnostic accuracy even when combined with echocardiography. Furthermore, neither test can confirm the diagnosis by itself or the type of amyloidosis. EMB remains the gold standard diagnostic method although clinically significant amyloidosis can be missed with small biopsies because of heterogeneous deposition. ${ }^{23}$

\section{Cardiac MRI and amyloidosis}

Several features are frequently found in CA: biventricular and atrial septal thickening, atrial enlargement, pleural and pericardial effusions, and diffuse myocardial LGE. There is great value in the diagnosis of CA by utilizing late gadolinium-enhanced cardiovascular magnetic resonance (LGE-CMR). Gadolinium chelates distribute in the extracellular space which is expanded by amyloid infiltration, and that leads to signal enhancement. The blood pool is dark in these patients, reflecting high tissue uptake and fast blood pool washout. ${ }^{24}$ Syed et al showed that LGE was present in a substantial proportion of patients with CA in whom LV thickness was normal by echocardiography. ${ }^{25}$ These findings suggest that cardiac tissue characterization by LGE may precede morphologic abnormalities. Furthermore, LGE in CA has different patterns; global LGE (transmural 'homogenous or heterogenous' or subendocardial), suboptimal nulling (SN) LGE (adequately nulled 'black' myocardium cannot be obtained), and patchy focal (PF) LGE. ${ }^{26}$ The most frequent pattern is global transmural or subendocardial LGE. ${ }^{25}$ The different patterns carry multiple outcomes, including symptoms, morphologic/functional variables, ECG voltages, and cardiac biomarker levels. The aforementioned are worst in patients with global diffuse LGE and trends downward in SN LGE, PF LGE, and absent LGE in that order. ${ }^{26}$

Despite the value of LGE in diagnosing CA, this method has some pitfalls: patchy subendocardial LGE early in the disease can lead to a false-negative LGE interpretation, and existing ischemic heart disease may affect LGE patterns on CMR. In a recent study, the diagnostic accuracy of LGE-CMR in detecting CA in patients undergoing EMB was as follows: sensitivity $88 \%$, specificity $90 \%$, positive predictive value $88 \%$, and negative predictive value $90 \%{ }^{27}$ Its accuracy may be underrepresented in cases of patchy cardiac involvement with negative EMB (sampling error) resulting in the LGE-CMR interpretation being classified as a false-positive.

\section{Conclusion}

Amyloidosis remains an underrecognized cause of cardiovascular disease. Despite multiple modalities for 
detection of amyloidosis, our patient had a negative fat biopsy as well as a negative bone marrow biopsy. The findings on cardiac MRI in this case were suggestive of amyloidosis. Subsequent genetic testing yielded a rare genetic mutation involving the TTR gene Ser23Asn. To the best of our knowledge, this is the second case ever reported. As such, the cardiac MRI is invaluable in shedding light on unexplained cardiomyopathy.

\section{Disclosure}

The authors report no conflicts of interest in this work.

\section{References}

1. Connors LH, Théberge R, Skare J, Costello CE, Falk RH, Skinner M. A new transthyretin variant (Ser23Asn) associated with familial amyloidosis in a Portuguese patient. Amyloid. 1999;6(2):114-118.

2. Kyle RA. Amyloidosis: the last three centuries. In: Bely M, Apathy A, editors. Amyloid and Amyloidosis. New York, NY: Springer; 2001:10-13.

3. Westermark P, Benson MD, Buxbaum JN, et al. Amyloid fibril protein nomenclature - 2002. Amyloid. 2002;9(3):197-200.

4. Rivera RJ, Vicenty S. Cardiac manifestations of amyloid disease. Bol Asoc Med PR. 2008;100(4):60-70.

5. Sunde M, Blake CC. From the globular to the fibrous state: protein structure and structural conversion in amyloid formation. $Q$ Rev Biophys. 1998;31(1):1-39.

6. Westermark P, Benson MD, Buxbaum JN, et al. A primer of amyloid nomenclature. Amyloid. 2007;14(3):179-183.

7. Sekijima Y, Wiseman RL, Matteson J, et al. The biological and chemical basis for tissue-selective amyloid disease. Cell. 2005;121(1):73-85.

8. Nakamura T, Higashi S, Tomoda K, Tsukano M, Baba S. Efficacy of etanercept in patients with AA amyloidosis secondary to rheumatoid arthritis. Clin Exp Rheumatol. 2007;25(4):518-522.

9. Merlini G, Bellotti V. Molecular mechanisms of amyloidosis. $N$ Engl J Med. 2003;349(6):583-596.

10. Hammarström P, Sekijima Y, White JT, et al. D18G transthyretin is monomeric, aggregation prone, and not detectable in plasma and cerebrospinal fluid: a prescription for central nervous system amyloidosis? Biochemistry. 2003;42(22):6656-6663.

11. Sekijima Y, Hammarstrom P, Matsumura M, et al. Energetic characteristics of the new transthyretin variant A25T may explain its atypical central nervous system pathology. Lab Invest. 2003;83(3): 409-417.
12. Mitsuhashi S, Yazaki M, Tokuda T, et al. Biochemical characteristics of variant transthyretins causing hereditary leptomeningeal amyloidosis. Amyloid. 2005;12(4):216-225.

13. Benson MD. The hereditary amyloidoses. Best Pract Res Clin Rheumatol. 2003;17(6):909-927.

14. Kyle RA, Gertz MA. Primary systemic amyloidosis: clinical and laboratory features in 474 cases. Semin Hematol. 1995;32(1): $45-59$.

15. Dubrey SW, Cha K, Skinner M, LaValley M, Falk RH. Familial and primary (AL) cardiac amyloidosis: echocardiographically similar diseases with distinctly different clinical outcomes. Heart. 1997;78(1): 74-82.

16. Hamilton JA, Benson MD. Transthyretin: a review from a structural prospective. Cell Mol Life Sci. 2001;58(10):1491-1521.

17. Mueller PS, Edwards WD, Gertz MA. Symptomatic ischemic heart disease resulting from obstructive intramural coronary amyloidosis Am J Med. 2000;109(3):181-188.

18. Al Suwaidi J, Velianou JL, Gertz MA, et al. Systemic amyloidosis presenting with angina pectoris. Ann Intern Med. 1999;131(11): $838-841$.

19. Chamarthi B, Dubrey SW, Cha K, Skinner M, Falk RH. Features and prognosis of exertional syncope in light-chain associated AL cardiac amyloidosis. Am J Cardiol. 1997;80(9):1242-1245.

20. Morner S, Hellman U, Suhr OB, Kazzam E, Waldenström A. Amyloid heart disease mimicking hypertrophic cardiomyopathy. J Intern Med. 2005;258(3):225-230.

21. Reisinger J, Dubrey SW, Lavalley M, Skinner M, Falk RH. Electrophysiologic abnormalities in AL (primary) amyloidosis with cardiac involvement. J Am Coll Cardiol. 1997;30(4):1046-1051.

22. Rahman JE, Helou EF, Gelzer-Bell R, et al. Noninvasive diagnosis of biopsy-proven cardiac amyloidosis. J Am Coll Cadiol. 2004;43(3): 410-415.

23. Falk RH. Diagnosis and management of the cardiac amyloidoses. Circulation. 2005;112(13):2047-2060.

24. Pennell DJ, Maceira AM. Magnetic resonance imaging in cardiac amyloidosis. JACC Cardiovasc Imaging. 2009;2(12):1378-1380.

25. Syed IS, Glockner JF, Feng D, et al. Role of cardiac magnetic resonance imaging in the detection of cardiac amyloidosis. JACC Cardiovasc Imaging. 2010;3(2):155-164.

26. Syed I, Glockner J, Araoz PA, et al. The presence and pattern of late gadolinium enhancement on cardiac MRI in amyloidosis is associated with other markers of prognosis. Circulation. 2008;118: S_1032.

27. Austin BA, Tang WH, Rodriguez ER, et al. Delayed hyper-enhancement magnetic resonance imaging provides incremental diagnostic and prognostic utility in suspected cardiac amyloidosis. JACC Cardiovasc Imaging. 2009;2(12):1369-1377.
Reports in Medical Imaging

\section{Publish your work in this journal}

Reports in Medical Imaging is an international, peer-reviewed, open access journal publishing original research, reports, reviews and commentaries on all areas of medical imaging. The manuscript management system is completely online and includes a very quick and fair peer-review system, which is all easy to use.

\section{Dovepress}

Visit http://www.dovepress.com/testimonials.php to read real quotes from published authors. 